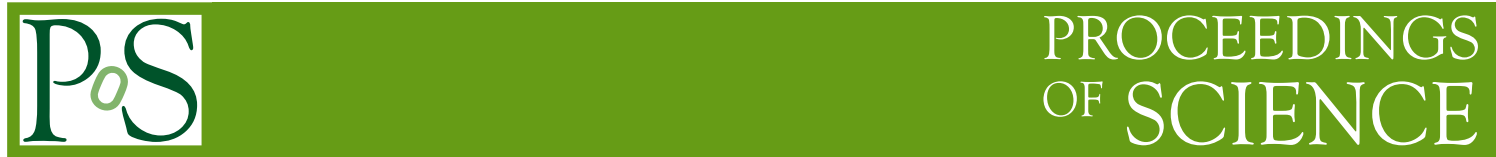

\title{
Effective spin models from the Yang-Mills action
}

\section{J. Greensite* \\ Niels Bohr International Academy, Blegdamsvej 17, DK 2100 Copenhagen Ø, Denmark \\ E-mail: greensitestars.sfsu.edu}

I present a method for finding the variation of the effective spin (or Polyakov line) action along any path in the configuration space of effective spins, given only the action of the underlying lattice gauge theory. The method can be applied, in principle, to extract the effective spin model from the underlying gauge theory.

Xth Quark Confinement and the Hadron Spectrum,

October 8-12, 2012

TUM Campus Garching, Munich, Germany

${ }^{*}$ Supported in part by the U.S. Department of Energy under Grant No. DE-FG03-92ER40711. 


\section{Introduction}

The study of the QCD phase diagram at large baryon density calls for the introduction of a quark chemical potential into the QCD Lagrangian. This results in an intrinsically complex action, with the consequence that importance sampling, essential to all lattice Monte Carlo simulations, can no longer be applied in a straightforward way. It is possible that one may get around the problem by simulating instead the corresponding Polyakov line action.

The Polyakov line action $S_{P}$, also known as the "effective spin" action, is obtained from the QCD action $S_{Q C D}$ by integrating out all degrees of freedom in the theory, under the constraint that the Polyakov line holonomies are fixed. This gives us, in principle, a $D=3$ dimensional theory whose phase structure ought to parallel that of full QCD. The integration can be carried out analytically at strong lattice couplings and heavy quark masses via the hopping parameter expansion, to give an action of the following form:

$$
S_{P}=\beta_{P} \sum_{\mathbf{x}} \sum_{i=1}^{3}\left[\operatorname{Tr} U_{\mathbf{x}}^{\dagger} \operatorname{Tr} U_{\mathbf{x}+\hat{i}}+\operatorname{Tr} U_{\mathbf{x}} \operatorname{Tr} U_{\mathbf{x}+\hat{i}}^{\dagger}\right]+\kappa \sum_{\mathbf{x}}\left[e^{\mu} \operatorname{Tr} U_{\mathbf{x}}+e^{-\mu} \operatorname{Tr} U_{\mathbf{x}}^{\dagger}\right]
$$

The sign problem in this model seems relatively mild, for a wide range of parameters $\beta_{P}, \kappa, \mu$, and can be handled in a number of different ways. These include the "flux representation" [1], reweighting [2], stochastic quantization [3], and mean field methods [4]. If we knew $S_{P}$ corresponding to $S_{Q C D}$ at realistic gauge couplings and light quark masses, and if the sign problem remains mild, then in principle we could determine the phase diagram of $S_{Q C D}$ by computing the phase diagram of $S_{P}$. The first problem, however, is to find $S_{P}$ in the parameter range of interest. In this talk I will discuss a novel approach to this problem; more details can be found in ref. [5]. ${ }^{1}$

\section{The relative weights approach}

Consider QCD on an $L^{3} \times N_{t}$ lattice volume, with $\mu=0$ for now. It is convenient to fix to temporal gauge, with timelike links set to the unit matrix everywhere except on one timeslice, say at $t=0$. Then, by definition

$$
\exp \left[S_{P}\left[U_{\mathbf{x}}\right]\right]=\int D U_{0}(\mathbf{x}, 0) D U_{k} D \bar{\psi} D \psi\left\{\prod_{\mathbf{x}} \delta\left[U_{\mathbf{x}}-U_{0}(\mathbf{x}, 0)\right]\right\} e^{S_{Q C D}} .
$$

Let us choose a set of "effective spin" configurations

$$
\left\{\left\{U_{\mathbf{x}}^{(i)}, \text { all } \mathbf{x} \in V_{3}\right\}, i=1,2, \ldots, M\right\} .
$$

where $V_{3}$ is a three-dimensional lattice of volume $L^{3}$, and imagine restricting the timelike link variables at $t=0$ to this set. Define the partition function

$$
\mathscr{Z}=\int D U_{0}(\mathbf{x}, 0) D U_{k} D \bar{\psi} D \psi \sum_{i=1}^{M}\left\{\prod_{\mathbf{x}} \delta\left[U_{\mathbf{x}}^{(i)}-U_{0}(\mathbf{x}, 0)\right]\right\} e^{S_{Q C D}},
$$

\footnotetext{
${ }^{1}$ For alternative approaches based on (i) strong-coupling expansions, cf. [2]; and on (ii) the inverse Monte Carlo method, cf. [6] and [7].
} 
and consider the ratio

$$
\begin{aligned}
& \frac{\exp \left[S_{P}\left[U^{(j)}\right]\right]}{\exp \left[S_{P}\left[U^{(k)}\right]\right]}=\frac{\int D U_{0}(\mathbf{x}, 0) D U_{k} D \bar{\psi} D \psi\left\{\prod_{\mathbf{x}} \delta\left[U_{\mathbf{x}}^{(j)}-U_{0}(\mathbf{x}, 0)\right]\right\} e^{S_{Q C D}}}{\int D U_{0}(\mathbf{x}, 0) D U_{k} D \bar{\psi} D \psi\left\{\prod_{\mathbf{x}} \delta\left[U_{\mathbf{x}}^{(k)}-U_{0}(\mathbf{x}, 0)\right]\right\} e^{S_{Q C D}}} \\
& =\frac{\frac{1}{\mathscr{Z}} \int D U_{0}(\mathbf{x}, 0) D U_{k} D \bar{\psi} D \psi\left\{\prod_{\mathbf{x}} \delta\left[U_{\mathbf{x}}^{(j)}-U_{0}(\mathbf{x}, 0)\right]\right\} e^{S_{Q C D}}}{\frac{1}{\mathscr{Z}} \int D U_{0}(\mathbf{x}, 0) D U_{k} D \bar{\psi} D \psi\left\{\prod_{\mathbf{x}} \delta\left[U_{\mathbf{x}}^{(k)}-U_{0}(\mathbf{x}, 0)\right]\right\} e^{S_{Q C D}}},
\end{aligned}
$$

Both the numerator and denominator have the interpretation of a probability, i.e.

$$
\operatorname{Prob}\left[U^{(j)}\right]=\frac{1}{\mathscr{Z}} \int D U_{0}(\mathbf{x}, 0) D U_{k} D \bar{\psi} D \psi\left\{\prod_{\mathbf{x}} \delta\left[U_{\mathbf{x}}^{(j)}-U_{0}(\mathbf{x}, 0)\right]\right\} e^{S_{Q C D}}
$$

is the probability that timelike links at $t=0$ are found to belong to the $j$-th member of the set (2.2).

Numerical simulation of the system described by $\mathscr{Z}$ is carried out by updating all degrees of freedom in the usual way, except for the timelike links at $t=0$. These timelike links are updated simultaneously, by choosing at random one of the $M$ members of the set (2.2), and accepting or rejecting it via the Metropolis algorithm. We keep track of the number of times $N_{n}$ that the $n$-th member of the set is accepted, out of the total $N_{t o t}=\sum_{n} N_{n}$. Then $\operatorname{Prob}\left[U^{(n)}\right]=N_{n} / N_{t o t}$, in the limit $N_{\text {tot }} \rightarrow \infty$, and therefore

$$
\frac{\exp \left[S_{P}\left[U^{(j)}\right]\right]}{\exp \left[S_{P}\left[U^{(k)}\right]\right]}=\lim _{N_{t o t} \rightarrow \infty} \frac{N_{j}}{N_{k}}
$$

From this information we can either test any proposal for $S_{P}$ or, possibly, determine $S_{P}$ from the data.

Let $\lambda$ parametrize a path $U_{\mathbf{x}}(\lambda)$ through the space of Polyakov line configurations. We can use (2.6) to compute path derivatives $d S_{P} / d \lambda$ at $\lambda=\lambda_{0}$. Let configuration $U_{\mathbf{x}}^{(j)}$ correspond to the point at $\lambda_{0}+\Delta \lambda$ and $U_{\mathbf{x}}^{(k)}$ to the point at $\lambda_{0}-\Delta \lambda$. Then it follows, from (2.6), that

$$
\left(\frac{d S_{P}\left[U_{\mathbf{x}}(\lambda)\right]}{d \lambda}\right)_{\lambda=\lambda_{0}} \approx \frac{1}{2 \Delta \lambda}\left\{\log \frac{N_{j}}{N_{\text {tot }}}-\log \frac{N_{k}}{N_{\text {tot }}}\right)
$$

Numerical accuracy can be improved by choosing a set of configurations $\left\{U_{\mathbf{x}}^{(n)}=U_{\mathbf{x}}\left(\lambda_{n}\right)\right\}$ along the path with

$$
\lambda_{n}=\lambda_{0}+\left(n-\frac{M+1}{2}\right) \Delta \lambda \quad, \quad n=1,2, \ldots, M
$$

Then for sufficiently small $\Delta \lambda$ we have

$$
\left(\frac{d S_{P}\left[U_{\mathbf{x}}(\lambda)\right]}{d \lambda}\right)_{\lambda=\lambda_{0}} \approx \text { slope of } \log \frac{N_{n}}{N_{t o t}} \text { vs. } \lambda_{n}
$$

Our long-term program is to first determine $S_{P}$ from $S_{Q C D}$ at $\mu=0$. Then we obtain $S_{P}$ at finite $\mu$ from the subsitution $U_{\mathbf{x}} \rightarrow e^{N_{t} \mu} U_{\mathbf{x}}, U_{\mathbf{x}}^{\dagger} \rightarrow e^{-N_{t} \mu} U_{\mathbf{x}}^{\dagger}$. From there, the idea is to solve the effective spin theory by any of the means (flux representation, reweighting, stochastic quantization, mean field) that have been suggested so far. The immediate goal, however, is to see if the relative weights method can extract $S_{P}$ from the underlying lattice gauge theory, and this talk will concentrate on the simplest example of pure $\mathrm{SU}(2)$ gauge theory. 


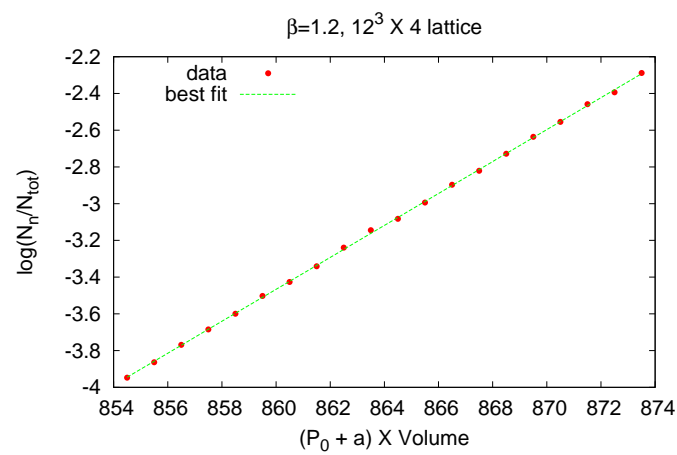

(a)

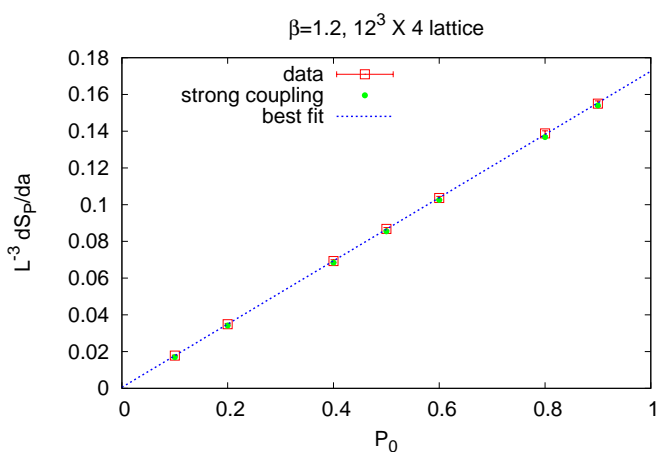

(b)

Figure 1: (a) The slope of the straight-line fit to the data shown gives an estimate for the derivative $L^{-3} d S_{P} / d a$ of $S_{P}$ with respect to the amplitude of spatially constant effective spin configurations. (b) A plot of the values for $L^{-3} d S_{P} / d a$ vs. $P_{0}$. Each data point is extracted from a plot similar to (a). Also shown are the corresponding strong-coupling values, and a best linear fit to the data points.

\section{A test at strong coupling}

The first question is whether the relative weights method can be used to determine $S_{P}$ in a case where the answer is known. Let us take $\beta=1.2, N_{t}=4$ in lattice $\mathrm{SU}(2)$ pure gauge theory. $S_{P}$ can then be readily computed by strong-coupling character expansion methods:

$$
S_{P}=\left[1+4 N_{t}\left(\frac{I_{2}(\beta)}{I_{1}(\beta)}\right)^{4}\right]\left(\frac{I_{2}(\beta)}{I_{1}(\beta)}\right)^{N_{t}} \sum_{\mathbf{x}} \sum_{i=1}^{3} \operatorname{Tr} U_{\mathbf{x}} \operatorname{Tr} U_{\mathbf{x}+\hat{i}}=\beta_{P} \sum_{\mathbf{x}} \sum_{i=1}^{3} P_{\mathbf{x}} P_{\mathbf{x}+\hat{i}}
$$

where $P_{\mathbf{x}} \equiv \frac{1}{2} \operatorname{Tr} U_{\mathbf{x}}$ and

$$
\beta_{P}=4\left[1+4 N_{t}\left(\frac{I_{2}(\beta)}{I_{1}(\beta)}\right)^{4}\right]\left(\frac{I_{2}(\beta)}{I_{1}(\beta)}\right)^{N_{t}} .
$$

The action can be divided into a potential and kinetic part

$$
\begin{aligned}
S_{P} & =K_{P}+V_{P} \\
K_{P} & =\frac{1}{2} \beta_{P} \sum_{\mathbf{x}} \sum_{i=1}^{3}\left(P_{\mathbf{x}} P_{\mathbf{x}+\hat{i}}-2 P_{\mathbf{x}}^{2}+P_{\mathbf{x}} P_{\mathbf{x}-\hat{i}}\right) \\
V_{P} & =3 \beta_{P} \sum_{\mathbf{x}} P_{\mathbf{x}}^{2},
\end{aligned}
$$

First we determine $V_{P}$ by relative weights. We choose the set of spatially constant configurations

$$
\begin{aligned}
U_{\mathbf{x}}^{(n)} & =\left(P_{0}+a_{n}\right) \mathbb{1}+i \sqrt{1-\left(P_{0}+a_{n}\right)^{2}} \sigma_{3} \\
a_{n} & =\left(n-\frac{1}{2}(M+1)\right) \Delta a, n=1,2, \ldots, M,
\end{aligned}
$$

So in this case $a$ is the $\lambda$ parameter, and it is easy to see that $d S_{P} / d a=d V_{P} / d P_{0}$. The data for $\log \left(N_{n} / N_{\text {tot }}\right)$ vs $L^{3}\left(P_{0}+a_{n}\right)$ at $P_{0}=0.5$ is shown in Fig. $1($ a), and the slope of this line gives us 


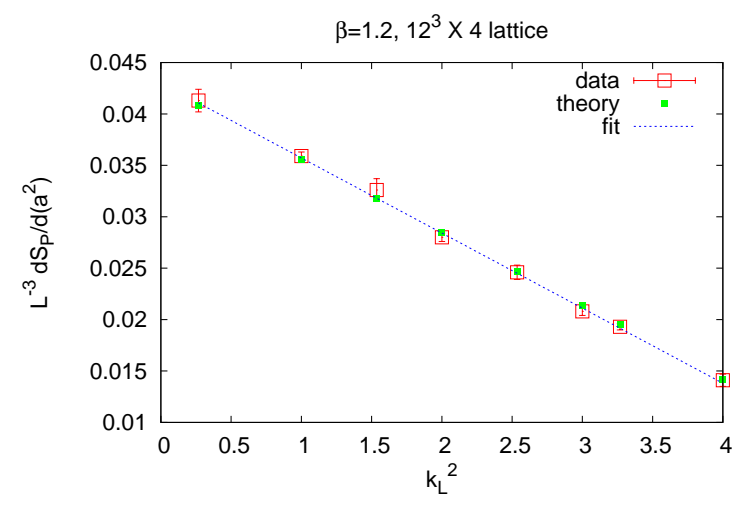

Figure 2: Derivative of the action w.r.t. path parameter $a^{2}$ vs. squared lattice momentum. Data is taken at strong gauge coupling $\beta=1.2$ for plane-wave deformations. Squares indicate the relative-weights values, while green dots are the values obtained from the strong-coupling expansion.

$L^{-3} d V_{P} / d P_{0}$. All data shown in this article was obtained at $L=12$. Repeating the calculation for various values of $P_{0}$ (Fig. 1(b)) we find that $d V_{P} / d P_{0}$ is linear in $P_{0}$. Integrating, and dropping an irrelevant constant of integration, the result is

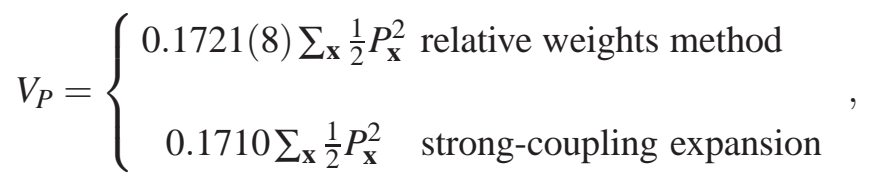

For the kinetic term, which by definition is zero for constant configurations, we choose a set of small plane wave deformations of a constant background

$$
\begin{aligned}
& U_{\mathbf{x}}^{(n)}=P_{\mathbf{x}}^{(n)} \mathbb{1}+i \sqrt{1-\left(P_{\mathbf{x}}^{(n)}\right)^{2}} \sigma_{3} \\
& P_{\mathbf{x}}^{(n)}=P_{0}+a_{n} \cos (\mathbf{k} \cdot \mathbf{x})
\end{aligned}
$$

where $k_{i}=2 \pi m_{i} / L$ are the components of the lattice wavevector, and it is convenient to choose $\lambda_{n}=a_{n}^{2}$ in this case. We compute $L^{-3} d S_{P} / d\left(a^{2}\right)$ from the slope of $\log \left(N_{n} / N_{\text {tot }}\right)$ vs $L^{3} a^{2}$, at fixed $\mathbf{k}$ and $P_{0}$, with the result, at fixed $P_{0}=0.5$ but different choices of $\mathbf{k}$, shown in Fig. 2, where $k_{L}^{2}$ is the usual (squared) lattice momentum $k_{L}^{2}=4 \sum_{i} \sin ^{2}\left(k_{i} / 2\right)$. It follows that $L^{-3} d S_{P} / d\left(a^{2}\right)=-A k_{L}^{2}+B$, and further simulations find that the constants $A, B$ are independent of $P_{0}$. This information, together with the knowledge of the potential, allows us to deduce the action $S_{P}$ (cf. [5] for details), and compare with the strong-coupling result:

$$
S_{P}=\left\{\begin{array}{cc}
.0292(8) \sum_{\mathbf{x}} \sum_{i=1}^{3} P_{\mathbf{x}} P_{\mathbf{x}+\hat{i}} & \text { (relative weights method) } \\
.0285 \sum_{\mathbf{x}} \sum_{i=1}^{3} P_{\mathbf{x}} P_{\mathbf{x}+\hat{i}} \quad \text { (strong-coupling expansion) }
\end{array}\right.
$$




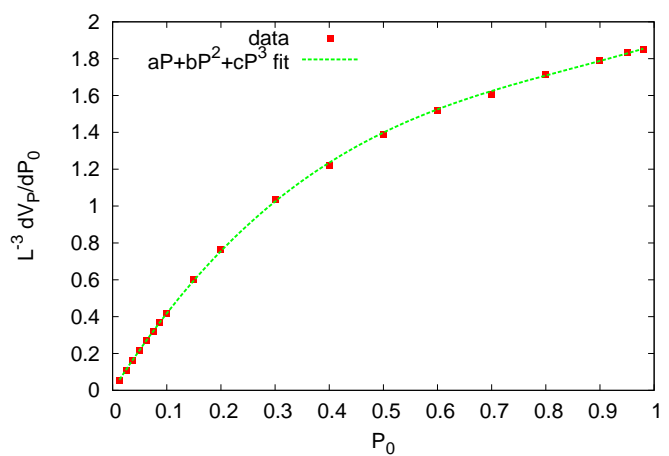

(a)

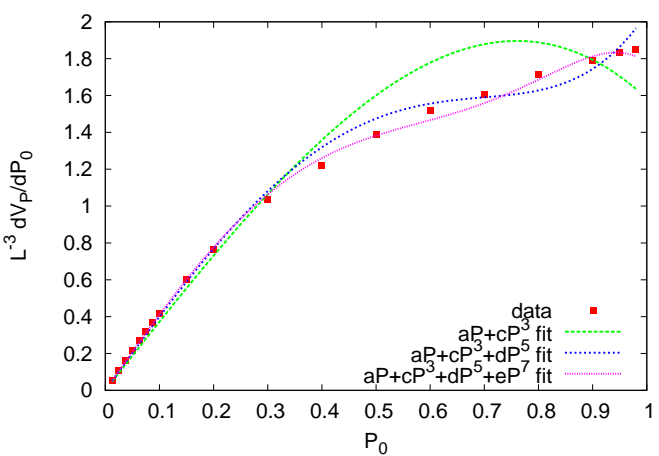

(b)

Figure 3: Derivatives of the potential. Subfigure (a) shows the best fit to the data by a polynomial $a P+b P^{2}+c P^{3}$, while subfigure (b) shows a best fit by polynomials with two, three, and four odd powers of $P$, which are forms that might be expected from unbroken center symmetry.

\section{The potential term at weaker couplings}

Our convention is that the potential term is local, while the kinetic term vanishes for spatially constant configurations $U$. In that case

$$
V_{P}=\sum_{\mathbf{x}} \mathscr{V}\left(U_{\mathbf{x}}\right) \quad \text { and } \quad \mathscr{V}(U)=\frac{1}{L^{3}} S_{P}(U)
$$

and $K_{P}=S_{P}-V_{P}$ by definition. To compute the potential defined in this way, we only need to compute the variation of $S_{P}$ with respect to the amplitude of spatially constant configurations. As at strong coupling, we choose the set (3.4) and compute $L^{-3} d S_{P} / d a=L^{-3} d V_{P} / P_{0}$, but this time we work at a weaker coupling $\beta=2.2, N_{t}=4$, which is still in the confined phase, and fit the result to a polynomial in $P_{0}$. The result is shown in Fig. 3(a), and the data is nicely fit by a cubic polynomial $c_{1} P_{0}+c_{2} P_{0}^{2}+c_{3} P_{0}^{3}$.

At first sight this result would seem to violate center symmetry, which for $\mathrm{SU}(2)$ requires that $V_{P}(P)=V_{P}(-P)$, and therefore the derivative should be an odd function of $P$, which would rule out the quadratic $c_{2} P_{0}^{2}$ term. On the other hand, fits to the data using only odd powers of $P_{0}$ are not very good, as we see in Fig. 3(b). The fit improves as more terms are added; it is, however, a little like fitting a step function with a truncated Fourier series. But in fact the quadratic term does not violate center symmetry, because simulations at $P_{0}<0$ find that the quadratic term is actually $c_{2} P_{0}^{2} \times \operatorname{sign}\left(P_{0}\right)$, and therefore, upon integration we find a center symmetric, albeit non-analytic, potential

$$
V_{P}=\sum_{\mathbf{x}}\left(\frac{1}{2} c_{1} P_{\mathbf{x}}^{2}+\frac{1}{3} c_{2}\left|P_{\mathbf{x}}\right|^{3}+\frac{1}{4} c_{3} P_{\mathbf{x}}^{4}\right)
$$

\section{Towards the kinetic term}

We consider two different sets of configurations. Type I will be small plane wave deformations on a constant background (3.6), as in the last section. Type II will simply be plane wave 
configurations

$$
\begin{aligned}
U_{\mathbf{x}}^{(n)} & =P_{\mathbf{x}}^{(n)} \mathbb{1}+i \sqrt{1-\left(P_{\mathbf{x}}^{(n)}\right)^{2}} \sigma_{3} \\
P_{\mathbf{x}}^{(n)} & =A_{n} \cos (\mathbf{k} \cdot \mathbf{x}) \\
A_{n} & =A_{0}+\left(n-\frac{1}{2}(M+1)\right) \Delta A, n=1,2, \ldots, M
\end{aligned}
$$

For Type I the $a$-dependence begins at $O\left(a^{2}\right)$ and we again compute $d S_{P} / d\left(a^{2}\right)$, but unlike the strong-coupling case the result does depend on $P_{0}$. For Type II configurations, we compute $d S / d A$.

It turns out that an expression for $S_{P}$ which seems to fit the data from Type I and Type II configurations has the form

$$
\begin{aligned}
S_{P}=2 c\left\{\sum_{\mathbf{x y}} P_{\mathbf{x}}\right. & \left.\left(\sqrt{-\nabla_{L}^{2}+g P_{a v}^{2}+g^{\prime} \Delta P^{2}}\right)_{\mathbf{x y}} P_{\mathbf{y}}-\sum_{\mathbf{x}} \sqrt{g P_{a v}^{2}+g^{\prime} \Delta P^{2}} P_{\mathbf{x}}^{2}\right\} \\
& +\sum_{\mathbf{x}}\left(\frac{1}{2} c_{1} P_{\mathbf{x}}^{2}+\frac{1}{3} c_{2}\left|P_{\mathbf{x}}^{3}\right|+\frac{1}{4} c_{3} P_{\mathbf{x}}^{4}\right)
\end{aligned}
$$

where

$$
P_{a v}=\frac{1}{L^{3}} \sum_{x} P_{x} \text { and } \Delta P^{2}=\frac{1}{L^{3}} \sum_{x}\left(P_{x}-P_{a v}\right)^{2}
$$

The data (red) and best fits (green) to the data for Type I and Type II configurations are shown in Figs. 4(a) and 4(b) respectively. The constants $c_{1}, c_{2}, c_{3}$ match the constants already determined for the potential. Although this action has been derived from data at very special (Type I and II) configurations, it is natural to conjecture, based on the success of this strategy at strong couplings, that (5.2) holds throughout the field configuration space.

\section{Conclusions}

The relative weights method for deriving the Polyakov line action has been tested at strong lattice couplings, where the answer known, and the method works well in that case. At a weaker coupling $\beta=2.2, N_{t}=4$ for pure $\mathrm{SU}(2)$ lattice gauge theory, where the Polyakov line action is not really known, a conjecture for the full action has been presented.

However, this conjecture comes with a strong caveat. The relative weights technique computes path derivatives of the Polyakov line action at any point along a path in the field configuration space, and the action $S_{P}$ has to be deduced from that information. In the present case these derivatives have been computed only at very special points in configuration space, and these points are quite atypical, when compared to the thermalized Polyakov line configurations that would be generated in the course of a standard lattice Monte Carlo finite temperature simulation. The approximate form that the action takes at such special points cannot be guaranteed to hold in the more general situation. Therefore it is crucial to test the proposed action in other, more typical, regions of field configuration space. If the form (5.2) of the action survives this test, then the next check would be to compute an observable such as the Polyakov line correlator, to see if it agrees with the same quantity computed in the underlying lattice gauge theory. I hope to report on the results of these studies in the near future. 


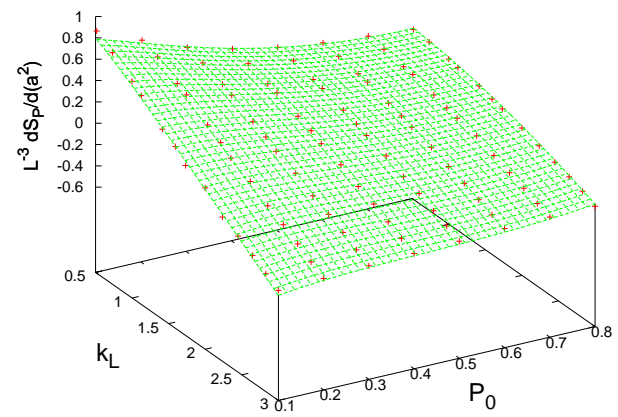

(a)

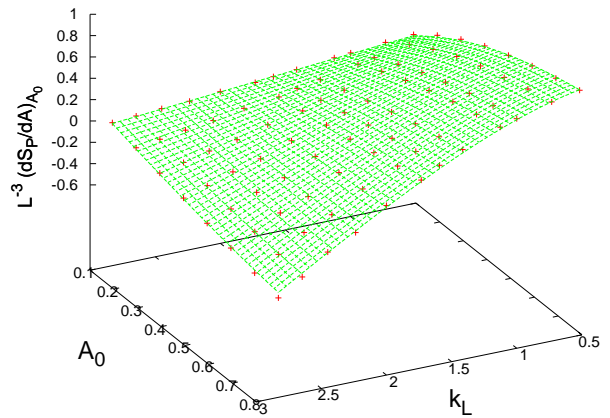

(b)

Figure 4: Derivatives of $S_{p}$ for Type I and Type II configurations. Red crosses are data points, and the green surface is a best fit to the data derived from the analytic form (5.2). (a) Type I configurations: $L^{-3} d S / d\left(a^{2}\right)$ vs. lattice momentum $k_{L}$ and Polyakov line $P_{0}$. (b) Type II configurations: Variation of the effective action with plane wave amplitude, $L^{-3} d S_{P} / d A$ evaluated at $A=A_{0}$, for Polyakov line configurations proportional to plane waves $P_{\mathbf{x}}=A \cos (\mathbf{k} \cdot \mathbf{x})$, as a function of $A_{0}$ and lattice momentum $k_{L}$.

\section{References}

[1] Y. D. Mercado and C. Gattringer, (2012), arXiv:1204.6074.

[2] M. Fromm, J. Langelage, S. Lottini, and O. Philipsen, JHEP 1201, 042 (2012), arXiv:1111.4953.

[3] G. Aarts and F. A. James, JHEP 1201, 118 (2012), arXiv:1112.4655.

[4] J. Greensite and K. Splittorff, Phys.Rev. D86, 074501 (2012), arXiv:1206.1159.

[5] J. Greensite, (2012), arXiv:1209.5697.

[6] L. Dittmann, T. Heinzl, and A. Wipf, JHEP 0406, 005 (2004), arXiv:hep-lat/0306032.

[7] T. Heinzl, T. Kaestner, and A. Wipf, Phys.Rev. D72, 065005 (2005), arXiv:hep-lat/0502013. 